\title{
Contracting singular cycles
}

\author{
by \\ Bernardo SAN MARTÍN * \\ Depto. de Matemáticas, Universidad Catolica del Norte, \\ Casilla 1280. Antofagasta-Chile. \\ E-mail: sanmarti@socompa.cecun.ucn.cl
}

\begin{abstract}
In the present paper we shall prove that the result in [2.2], concerning the measure of the bifurcating set for generic one-parameter families through a simple contracting singular cycle with only one periodic orbit, is still true for cycles with any number (finitely many) periodic orbits. The novelty is the choice of a proper sections to the flow and the corresponding Poincare maps.

(C) 1998 L'Association Publications de l'Institut Henri Poincaré. Published by Elsevier B.V. All rights reserved

RÉSuMÉ. - Dans cet article nous montrons que le résultat dans [2.2] sur la mesure de bifurcation pour des familles génériques à 1 paramètre à travers un simple cycle singulier contractant avec une orbite périodique est vrai aussi pour des cycles avec tout nombre (fini) d'orbites périodiques. La nouveauté réside dans le choix des sections appropriées du flot et des applications de Poincaré correspondantes.

(C) 1998 L'Association Publications de l'Institut Henri Poincaré. Published by Elsevier B.V. All rights reserved
\end{abstract}

\section{INTRODUCTION}

In this work we continue the analysis of singular cycles started in [1] and continued in [2]. Recall that a singular cycle for a vector field is a finite set of singularities and periodic orbits, all of them hyperbolic, linked in a

* 1991 Mathematics Suject Classifications. $58 \mathrm{f} 14,58 \mathrm{f} 1258 \mathrm{f} 13$. Partially supported by CNPq (Brazil) and FONDECYT 1941080 (Chile).

Annales de l'Institut Henri Poincaré - Analyse non linéaire - 0294-1449 Vol. $15 / 98 / 05 /$ 
cyclic way by orbits in the intersections of the stable and unstable manifolds of the singularities and periodic orbits. Moreover, if the cycle have only one singularity and the expanding eigenvalue is smaller than the weakest contracting one, we call it contracting. Otherwise, we call it expanding. In [2], it was proved that in the unfolding of a contracting singular cycle with only one periodic orbit satisfying certain additional conditions, which holds for a large class of these fields, leads to the creation of at most onc attracting periodic orbit. Moreover, the set of parameters corresponding to hyperbolic dynamics in the parameter space has full Lebesgue measure.

Now we will give the precise description of the problem and the results in [1] and [2]. Let $\mathrm{M}$ be a compact and boundaryless 3-manifold and let $\mathcal{X}^{r}$ be the Banach space of $C^{r}$ vector fields on M. If $X \in \mathcal{X}^{\prime r}$, denote by $\Gamma(X)$ its chain recurrent set. We say that $X$ is simple when $\Gamma(X)$ is a union of finitely many hyperbolic critical orbits. By critical orbit we mean an orbit that is either periodic or singular. It is easy to see that the set $S^{r}$ of simple $C^{r}$ vector fields is an open subset on $\mathcal{X}^{r}$.

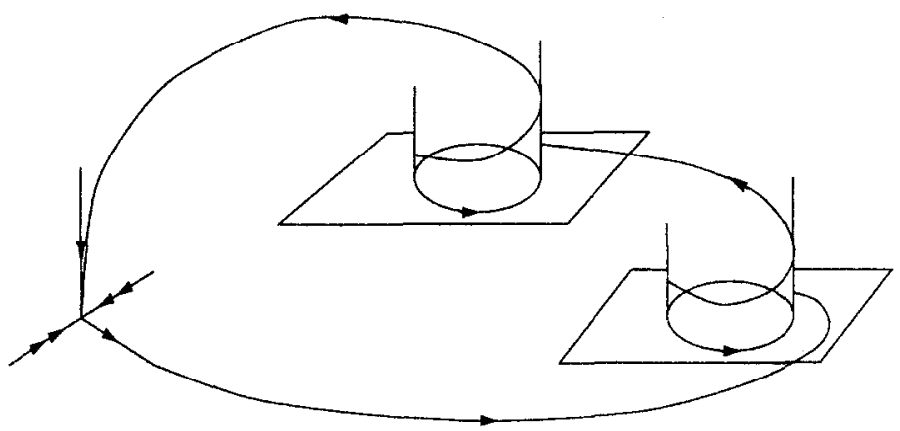

Fig. 1.

Our objet of study will be simple singular cycles. That is, a cycle $\Lambda$ of $X \in \mathcal{X}^{r}$ satisfying:

$-\Lambda$ contains a unique singularity $\sigma_{0}$;

- the eigenvalues of $D_{\sigma_{0}} X: T_{\sigma_{0}} M \hookleftarrow$ are real and satisfy $-\lambda_{3}<$ $-\lambda_{1}<0<\lambda_{2}$

- $\Lambda$ contains a unique non singular orbit $\gamma_{0}$ contained in $W^{u}\left(\sigma_{0}\right)$ and such that $\omega-\lim \left(\gamma_{0}\right)$ is a periodic orbit $\sigma_{1}$ and the other regular orbit are transversal intersection of stable and unstable manifolds;

- for each $p \in \gamma_{0}$ and each invariant manifold $W\left(\sigma_{0}\right)$ of $X$ passing through $\sigma_{0}$ and tangent at $\sigma_{0}$ to the eigenspace spanned by the 
eigenvectors associated to $-\lambda_{1}$ and $\lambda_{2}$, we have

$$
T_{p} W\left(\sigma_{0}\right)+T_{p} W^{s}\left(\sigma_{1}\right)=T_{p} M
$$

- there exist neighborhood $\mathcal{U}$ of $X$ such that if $Y \in \mathcal{U}$ the continuations $\sigma_{i}(Y), i: 0, . ., k$ of the critical orbits $\sigma_{i}$ of the cycle are well defined and the vector field $Y$ is $C^{2}$-linearizable in $\sigma_{0}$ as well as the Poincare maps of $\sigma_{i}(Y), i: 1, . ., k$;

$-\Lambda$ is isolated. Here, this mean that there exist a neighborhood $U$ of $\Lambda$, called isolating block, such that $\prod_{t} X_{t}(U)$ no contains orbit close $\gamma_{0}$, where $X_{t}: M \hookleftarrow$ is the flow generated by $X$.

For simple singular cycles the following result holds [1]:

THEOREM 1. - Let $\Lambda_{0}$ be a simple singular cycle for a vector field $X_{0}$, and let $U$ be an isolating block of $\Lambda_{0}$. Then there are a neighborhood $\mathcal{U}$ of $X_{0}$ and a co-dimension one submanifold, $\mathcal{N} \subset \mathcal{X}^{r}$ containing $X_{0}$ such that

- If $Y \in \mathcal{U} \cap \mathcal{N}$, then $\Lambda(Y, U)=\bigcap_{t} Y_{t}(U)$ contains a singular cycle topologically equivalent to $\Lambda_{0}$

$-\mathcal{U} \backslash \mathcal{N}$ has two connected components and one of them denoted by $\mathcal{U}^{-}$is such that $Y \in \mathcal{U}^{-}$implies that the chain recurrent set of $Y / \Lambda(Y, U)$ consists of the continuations $\sigma_{i}(Y), 0 \leq i \leq k$ of the critical orbits $\sigma_{i}$ of $\Lambda_{0}$.

This means that the cycle persists in $\mathcal{N} \cap \mathcal{U}$ and breaks down in $\mathcal{U}^{-}$. Denote by $\mathcal{U}^{+}$the other component of $\mathcal{U} \backslash \mathcal{N}$. Define $\mathcal{U}_{H}^{+}$as the set of $Y \in \mathcal{U}^{+}$such that the chain recurrent set for $Y$ in $\Lambda(Y, U)$, consists of $\sigma_{0}(Y)$ plus a transitive hyperbolic set. Also let $\mathcal{U}_{H^{\prime}}^{+}$be the set of $Y \in \mathcal{U}^{\prime}$ such that the recurrent set of $Y$ in $U$ consists of the union of $\sigma_{0}(Y)$, a transitive hyperbolic set, and a unique attracting periodic orbit.

The study of $\mathcal{U}^{+} \backslash\left(\mathcal{U}_{H}^{+} \cap \mathcal{U}_{H^{\prime}}^{+}\right)$depends on eigenvalues at the singularity $\sigma_{0}$ of the cycle. If $\lambda_{1}-\lambda_{2}<0$, i.e. the expanding case, it was proved in [1] that $\mathcal{U}_{H}^{+}$, is empty and $\mathcal{U}_{H}^{+}$is dense in $\mathcal{U}^{+}$. In the contracting case, namely $\lambda_{1}-\lambda_{2}>0$, we have the following result for cycles with only one periodic orbit and eigenvalues such that $\beta>\alpha+2$, where $\beta=\frac{\lambda_{3}}{\lambda_{2}}$ and $\alpha=\frac{\lambda_{1}}{\lambda_{2}}$. This case is called strongly contracting.

THEOREM 2. - ([2]) If $X_{\mu}$ is a family transversal to $\mathcal{N}$ at $X_{0}$ and $X_{0}$ has a strongly contracting simple singular cycle with only one periodic orbit, then for some $t>0$

$$
m\left\{0 \leq \mu \leq t: X_{\mu} \in \mathcal{U}^{+} \backslash\left(\mathcal{U}_{H}^{+} \cap \mathcal{U}_{H^{\prime}}^{+}\right)\right\}=0
$$

Here, the condition $\beta>\alpha+2$ was used in [2] to ensure the existence of a $C^{3}$-stable foliation associated to the cycle $\Lambda_{0}$. So, the dynamics in the space Vol. $15, n^{\circ} 5-1998$. 
of leaves of this foliation is given by a one-dimensional mapping which has negative Shwarzian derivative. Ilowever, it is enough to have a $C^{2}$ foliation, which exists if $\beta>\alpha+1$, and to use that such one-dimensional mapping has monotonic derivative.

THEOREM 3. - If $X_{\mu}$ crosses transversally at $\mathcal{N}$ in $X_{0}$ and $X_{0}$ has simple singular cycle $\Lambda_{0}$ such that $\beta>\alpha+1$, then for some $t>0$

$$
m\left\{0 \leq \mu \leq t: X_{\mu} \in \mathcal{U} \backslash\left(\mathcal{U}_{H}^{+} \cap \mathcal{U}_{H^{\prime}}^{+}\right)\right\}=0
$$

\section{PROOF THEOREM 3}

The method of proof is essentially the same used in [2]. So, we only need to show that lemma 1 there is still valid for the maps $f_{\mu}$ that we define below (see lemma 2).

Let $X_{\mu}$ be a family as in Theorem 3. We assume that the cycle contains two periodic orbits $\sigma_{1}$ and $\sigma_{2}$.

Let $S_{1}$ and $S_{2}$ be cross sections to the flow $X_{0}$ at $q_{1} \in \sigma_{1}$ and at $q_{2} \in \sigma_{2}$ parametrized by linear coordinates $\{(x, y):|x|,|y| \leq 1\}$ and satisfying $W^{s}\left(\sigma_{i}\right) \supseteq\{(x, 0):|x| \leq 1\}$ and $W^{u}\left(\sigma_{i}\right) \supseteq\{(0, y):|y| \leq 1\}$.

A closed subset $C \subset S_{i}$ is called horizontal strip if it is bounded in $S_{i}$ by two disjoint continuous curves connecting the vertical sides of $S_{i}$.

Since $W^{u}\left(\sigma_{2}\right)$ intersects $W^{s}\left(\sigma_{0}\right)$ and $\gamma_{0}$ has as $\omega$-limit set $\sigma_{1}$, there is a horizontal strip $R_{4} \subset S_{2}$ such that the Poincare map $F_{0}: R_{4} \rightarrow S_{1}$ is well defined. Clearly $F_{0}$ is defined in two horizontal strips $R_{1} \subset S_{1}$ and $R_{3} \subset S_{2}$ and coincides with the Poincare maps associated to the periodic orbits $\sigma_{1}$ and $\sigma_{2}$ respectively. Moreover, there is also a region $R_{2} \subset S_{1}$ such that the Poincare map is defined from $R_{2}$ into $R_{3}$ (see Fig. 2).

Finally for $\mu>0$ sufficiently small, $S_{i}$ is still a cross section for $X_{\mu}$ at $\sigma_{i}(\mu)$ and $W^{u}\left(\sigma_{0}(\mu)\right)$ intersects $S_{1}$ at $p_{\mu}=\left(x_{\mu}, y_{\mu}\right)$, where $\sigma_{i}(\mu)$ are the continuations of $\sigma_{i}$. As before, the Poincare map $F_{\mu}$ is defined on $R_{1}(\mu) \cup R_{2}(\mu) \cup R_{3}(\mu) \cup R_{4}(\mu)$ and the restrictions to $R_{1}(\mu)$ and $R_{3}(\mu)$ coincide with the Poincare maps $P_{\sigma_{1}(\mu)}$ and $P_{\sigma_{2}(\mu)}$ associated to the periodic orbits $\sigma_{1}(\mu)$ and $\sigma_{2}(\mu)$ respectively.

Up to replacing $R_{2}(\mu)$ by some negative iterate of it and $R_{3}(\mu)$ by some positive iterate, we may assume that the horizontal lines are very contracted and the verticals ones are very extended. Now, applying this fact and the same techniques as in [1] and [3], one proves the following lemma. 


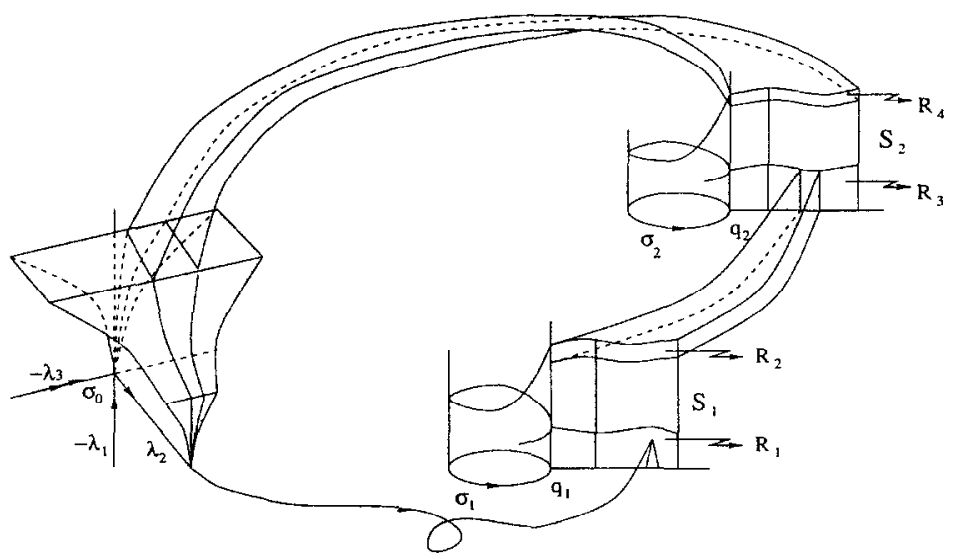

Fig. 2.

LEMMA 1. - If $\beta>\alpha+1$, then for every $\mu \geq 0$ sufficiently small there is an invariant $C^{2}$-stable foliation $\mathcal{F}_{\mu}^{s}$ by $F_{\mu}$ depending $C^{1}$ on $\mu$.

Let $\left\{f_{\mu}: \mathcal{D}\left(f_{\mu}\right) \subset[0,1] \cup[2,3]: \mu \geq 0\right\}$ be the family of one-dimensional map induced by $\left\{\mathcal{F}_{\mu}^{s}: \mu \geq 0\right\}$. Since $\mathcal{F}_{\mu}^{s}$ is $C^{2}$ each $f_{\mu}$ is also $C^{2}$, and it depends $C^{1}$ on $\mu$. We can parametrize $\left\{f_{\mu}\right\}$ in such a way that $f_{\mu}(3)=\mu$. It is easy to see that the general form of $f_{\mu}$ is:

$$
f_{\mu}(x)= \begin{cases}\rho_{\mu} \cdot x & x \in\left[0, \rho_{\mu}^{-1}\right] \\ g_{\mu}(x) & x \in[a, 1] \\ \sigma_{\mu} \cdot(x-2)+2 & x \in\left[2,2+\sigma_{\mu}^{-1}\right] \\ \mu-K_{\mu}(x) \cdot(3-x)^{\alpha_{\mu}} & x \in\left[a_{\mu}, 3\right]\end{cases}
$$

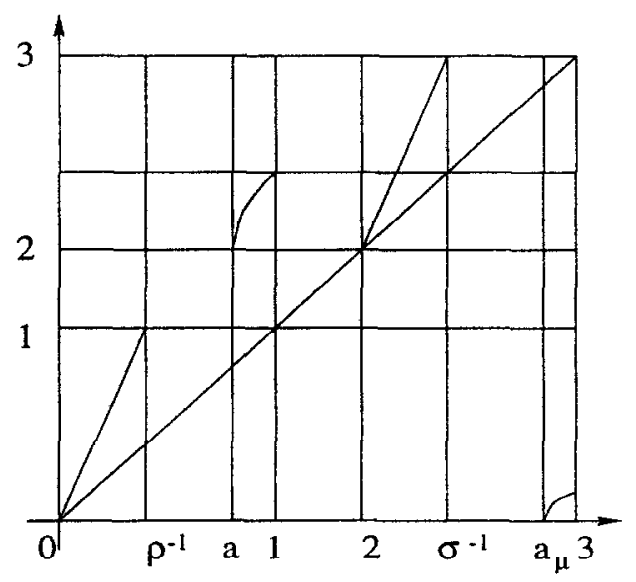

Fig. 3.

Vol. 15, n 5-1998. 


\section{Remarks}

a. - Actually the function $g_{\mu}$ is defined on one interval $\left[a, b_{\mu}\right]$ with $b_{\mu}$ close to 1 , but without loss of generality we can assume that $b_{\mu}$ is equal to 1 .

b. $-\rho_{\mu}$ is the expanding eigenvalue of the Poincare map associated to $\sigma_{1}(\mu)$, and $\sigma_{\mu}$ is the corresponding one for $\sigma_{2}(\mu)$.

c. $-K_{\mu}$ is $C^{2}$ and $K_{\mu}(3)>0$.

d. - We may choose $a$ close to 1 such that $\frac{4}{\rho_{\mu}} \cdot \frac{1-a}{a-\rho_{\mu}^{-1}}<\frac{1}{2}$ and $\left|g_{\mu}^{\prime}(x)\right|>1$ for all $x \in[a, 1]$.

e. - Since $f_{\mu}\left(a_{\mu}\right)=0$ we have $a_{\mu} \rightarrow 3$ when $\mu \rightarrow 0$.

f. - We will suppose that $\int_{\mu}$ is increasing.

The following properties of $f_{\mu}$ can be easily verified.

There exists $\bar{\mu}>0$ such that for every $\mu \in[0, \bar{\mu}]$ we have:

i. $-\left|\frac{\partial}{\partial \mu} f_{\mu}(x)-1\right|<\epsilon$ for all $x \in\left[a_{\mu}, 3\right]$ and small $\epsilon$.

ii. $-0<f_{\mu}^{\prime}(x)<\epsilon$ for all $x \in I_{4}$.

iii. - For $f_{\mu}^{m}: I_{2} \rightarrow I_{2}$, where this map has sense, $\left(f_{\mu}^{m}\right)^{\prime \prime}$ is negative, which implies that $\left(f_{\mu}^{m}\right)^{\prime}$ is decreasing.

Without loss of generality we will assume $\rho_{\mu}=\rho$ and $\sigma_{\mu}=\sigma$. Let $I_{1}=\left[0, \rho^{-1}\right], I_{2}=[a, 1], I_{3}=\left[2,2+\sigma^{-1}\right]$ and $I_{4}(\mu)=\left[a_{\mu}, 3\right]$. Define $\Delta_{\mu}=\left\{x: f_{\mu}^{n}(x) \in \cup_{i=1}^{4} I_{i}(\mu) \forall n \geq 0\right\}, \dot{\Delta}=\left\{\mu: 3 \notin \Delta_{\mu}\right\}$, and $H=\left\{\mu: f_{\mu}\right.$ is hyperbolic $\}$

We now claim that

1) if $\mu \in \tilde{\Delta}$, then $\mu \in H$

2) if $f_{\mu}$ has a hyperbolic attracting periodic orbit in $\Delta_{\mu}$, then $\mu \in H$.

In fact, from (iii) we have that there exists at most one attracting periodic orbit in $I_{2}$ (Singer's theorem [4]) which attracts the critical point. Then, in both cases all periodic points are hyperbolic. Therefore, by Mañe's theorem [5], we have that $f_{\mu}$ is hyperbolic.

Denote by $m$ the Lebesgue measure. Theorem 3 is a consequence of the following theorem.

THEOREM 4. - There exists $\bar{\mu}>0$ such that $m(H \cap[0, \bar{\mu}])=\bar{\mu}$.

To obtain this result we will prove that there exists $\bar{\mu}>0$ such that for every $\mu_{0} \in[0, \bar{\mu}]$,

$$
\lim _{\epsilon \rightarrow 0} \frac{\left(H^{c} \cap\left[\mu_{0}-\epsilon, \mu_{0}+\epsilon\right]\right)}{2 \epsilon}<1
$$

where $H^{c}$ is the complement of $H$ in $\mathbb{R}$. 
Suppose that $f_{\mu}^{j}(3) \in \cup_{i=1}^{4} I_{i}(\mu), 0 \leq j \leq N$. Define the sequence $\xi_{N}(\mu)$ by

$$
\xi_{N}(\mu)(j)=i \quad \text { if } \quad f_{\mu}^{j}(3) \in I_{i}(\mu) ; \quad 0 \leq j \leq N
$$

Lemma 2. - Suppose that $\xi_{N}(\mu)$ is defined and constant on the interval $\left[\mu_{0}, \mu_{1}\right]$. Then, there exists $M>0$ such that $\mathcal{X}_{n}(\mu) \leq M \mathcal{X}_{n}\left(\mu_{0}\right)$ for every $\mu \in\left[\mu_{0}, \mu_{1}\right]$ where $\mathcal{X}_{n}(\mu)=\partial_{\mu} f_{\mu}^{n}(3)$.

This lemma follows immediately from

LEMMA 3. - Under the above conditions. If $f_{\mu}^{n}(3) \in I_{1} \cup I_{4}(\mu)$, then

$$
\frac{\mathcal{X}_{n+1}(\mu)}{\mathcal{X}_{n+1}\left(\mu_{0}\right)} \leq 2
$$

Proof. - Firstly, observe that $\mathcal{X}_{n}(\mu)>\frac{1}{2}$ for every $n \leq N$. In fact

$$
\begin{array}{ll}
\mathcal{X}_{n+1}(\mu)=\rho \mathcal{X}_{n}(\mu) & f_{\mu}^{n}(3) \in I_{1} \\
\mathcal{X}_{n+1}(\mu)=g_{\mu}^{\prime}\left(f_{\mu}^{n}(3)\right) \mathcal{X}_{n}(\mu)+\left.\partial_{\mu} g_{\mu}(x)\right|_{x=f_{\mu}^{n}(3)} & f_{\mu}^{n}(3) \in I_{2} \\
\mathcal{X}_{n+1}(\mu)=\sigma \mathcal{X}_{n}(\mu) & f_{\mu}^{n}(3) \in I_{3} \\
\mathcal{X}_{n+1}(\mu)=f_{\mu}^{\prime}\left(f_{\mu}^{n}(3)\right) \mathcal{X}(\mu)+\left.\partial_{\mu} f_{\mu}(x)\right|_{x=f_{\mu}^{n}(3)} & f_{\mu}^{n}(3) \in I_{4}
\end{array}
$$

Hence, using the properties of $f_{\mu}$ the assertion follows by inductions. Since, if $f_{\mu}^{n}(3) \in I_{2}$ then $\frac{\left.\partial_{\mu} f_{\mu}(x)\right|_{x=f_{\mu}^{n}(3)}}{\mathcal{X}_{n}(\mu)}$ is small for $\mu$ small enough, lemma 2 it follows.

The hypotheses of lemma implies that

$$
f_{\mu}^{n}(3)-f_{\mu_{0}}^{n}(3) \geq \frac{1}{2}\left(\mu-\mu_{0}\right)
$$

Now we prove the lemma 2 by induction.

1. - If $f_{\mu}^{n}(3) \in I_{1}$ then $f_{\mu}^{n+1}(3)=\rho f_{\mu}^{n}(3)$. Hence we have

$$
\frac{\mathcal{X}_{n+1}(\mu)}{\mathcal{X}_{n+1}\left(\mu_{0}\right)} \leq 2 \text {. }
$$

2.- If $f_{\mu}^{n}(3) \in I_{4}$ then $f_{\mu}^{n}(3)=f_{\mu}^{n_{0}+1+k+1}(3)$ where $f_{\mu}^{n_{0}}(3) \in I_{1}$ and $f_{\mu}^{n_{0}+1}(3) \in I_{2}$. At once $f_{\mu}^{n+1}(3)=f_{\mu}\left(h_{\mu}\left(f_{\mu}^{n_{0}+1}(3)\right)\right)$ where $h_{\mu}=$ $\sigma^{k}\left(g_{\mu}-2\right)+2$. Thus we obtain

$$
\begin{aligned}
\mathcal{X}_{n+1}(\mu)= & \partial_{\mu} f_{\mu}\left(h_{\mu}\left(f_{\mu}^{n_{0}+1}(3)\right)\right)+\partial_{x} f_{\mu}\left(h_{\mu}\left(f_{\mu}^{n_{0}+1}(3)\right)\right) \partial_{\mu} h_{\mu}\left(f_{\mu}^{n_{0}+1}(3)\right)+ \\
& \partial_{x} f_{\mu}\left(h_{\mu}\left(f_{\mu}^{n_{0}+1}(3)\right)\right) \partial_{x} h_{\mu}\left(f_{\mu}^{n_{0}+1}(3)\right) \mathcal{X}_{n_{0}+1}(\mu) .
\end{aligned}
$$


Now we claim that

a) $\partial_{\mu} f_{\mu}\left(h_{\mu}\left(f_{\mu}^{n_{0}+1}(3)\right)\right)+\partial_{x} f_{\mu}\left(h_{\mu}\left(f_{\mu}^{n_{0}+1}(3)\right)\right) \partial_{\mu} h_{\mu}\left(f_{\mu}^{n_{0}+1}(3)\right)$

$$
\leq\left. 2\left[\partial_{\mu} f_{\mu}\left(h_{\mu}\left(f_{\mu}^{n_{0}+1}(3)\right)\right)+\partial_{x} f_{\mu}\left(h_{\mu}\left(f_{\mu}^{n_{0}+1}(3)\right)\right) \partial_{\mu} h_{\mu}\left(f_{\mu}^{n_{0}+1}(3)\right)\right]\right|_{\mu=\mu_{0}}
$$

b) $\partial_{x} f_{\mu}\left(h_{\mu}\left(f_{\mu}^{n_{0}+1}(3)\right)\right) \partial_{x} h_{\mu}\left(f_{\mu}^{n_{0}+1}(3)\right)$

$$
\leq \partial_{x} f_{\mu_{0}}\left(h_{\mu_{0}}\left(f_{\mu_{0}}^{n_{0}+1}(3)\right)\right) \partial_{x} h_{\mu_{0}}\left(f_{\mu_{0}}^{n_{0}+1}(3)\right)
$$

Then the lemma follows by induction.

Proof of (a):

Let $x_{0}=f_{\mu_{0}}^{n_{0}+1}(3), x_{1}=f_{\mu}^{n_{0}+1}(3) ; x_{0}, x_{1} \in I_{2}, y_{0}=h_{\mu_{0}}\left(x_{0}\right)$, $y_{1}=h_{\mu}\left(x_{1}\right) ; y_{0}, y_{1} \in I_{4}$

So using $\mathbf{i}$ we have that

$$
\left.2 \partial_{\mu} f_{\mu}\left(y_{0}\right)\right|_{\mu=\mu_{0}}-\partial_{\mu} f_{\mu}\left(y_{1}\right) \approx 1
$$

On the other hand, $\partial_{\mu} g_{\mu}(a)=0$ we have that for every $x \in I_{2}$ such that $f_{\mu}^{k}\left(g_{\mu}(x)\right)=y \in I_{4}$

$$
\partial_{\mu} g_{\mu}(x)=\partial_{\mu, x}^{2} g_{\mu}(z)\left[\partial_{x} g_{\mu}(\tilde{z})\right]^{-1} \sigma^{-k}(y-2)
$$

where $z, \tilde{z} \in I_{2}$

Thus $\left|\partial_{\mu} h_{\mu}(x)\right|=\left|\sigma^{k} \partial_{\mu} g_{\mu}\right| \leq C, C$ independing of $k$. From here and ii we obtain that

$$
\partial_{x} f_{\mu}(y) \partial_{\mu} h_{\mu}(x)-\left.2\left[\partial_{x} f_{\mu_{0}}\left(y_{0}\right) \partial_{\mu} h_{\mu}\left(x_{0}\right)\right]\right|_{\mu=\mu_{0}} \approx 0
$$

Thus (a) is proved.

Proof of (b):

Let $F(\mu, x)=\partial_{x}\left(f_{\mu} \circ h_{\mu}\right)(x)$. In order to have (b) is sufficient to prove that $D F_{(\mu, x)}(1, v)<0$ for all $v>\frac{1}{2}$. This is clear since

$$
D F_{(\mu, x)}(1, v)=\sigma^{2 k}(3-\tilde{x})^{\alpha-2}\left[(3-\tilde{x}) M_{k}(\mu, x)+L_{k}(\mu, x) v\right]
$$

and $L_{k}(\mu, x) \leq c<0, \mathrm{c}$ is independent of $k, M_{k}$ are uniformly bounded in $k$ and $\tilde{x}=f_{\mu}^{k}\left(g_{\mu}(x)\right) \in I_{4}$.

To finish the proof of the theorem we can follow [2]. 


\section{ACKNOWLEDGEMENTS}

The author would like to express his grateful to Prof. J Palis Jr. for all the comments during the preparation of the present paper. Also want to express his acknowledgments to $\mathrm{C}$. Morales and I. Huerta by all the fruitful conversations related with this work.

\section{REFERENCES}

[1] R. Bamon, R. Labarca, R. Mañe and M. J. Pacifico, The explosion of singular cycles. I.H.E.S., Publications Mathématiques, Vol. 78, 1993, pp. 207-232.

[2] M. J. Pacifico and A. Rovella, Unfolding contracting cycles. Amn. Scient. Ec. Norm. Sup., $4^{e}$ serie t.26, 1993, pp. 691-700.

[3] A. Rovella, The Dynamics of Perturbations of the contracting Lorenz attractor, Bol. Soc. Bras. Math., vol 24, N. 2, 1993, pp. 233-259.

[4] S. Singer, Stable orbits and bifurcations of map of the interval, SIAM J. Appl. Math., Vol. 35, 1978, pp. 260-267.

[5] R. MAÑE, Hyperbolicity, sinks and measure in one dimensional dynamics, Comm. Math. Phys., Vol. 100, 1985, pp. 495-524 and Erratum, Comm. Math. Phys., Vol. 112, 1987, pp. 721-724.

(Manuscript received July 10, 1996.)

Vol. $15, n^{\circ} 5-1998$. 